\title{
Non-homogeneous strongly singular integrals
}

\author{
by
}

\section{Bassam Shayya (Beirut)}

\begin{abstract}
We study the $L^{p}$ mapping properties of a family of strongly singular oscillatory integral operators on $\mathbb{R}^{n}$ which are non-homogeneous in the sense that their kernels have isotropic oscillations but non-isotropic singularities.
\end{abstract}

1. Introduction. Let $n \geq 2,1=b_{1} \leq b_{2} \leq \cdots \leq b_{n}$ be given constants, and for $x \in \mathbb{R}^{n}$ define

$$
\tau(x)=\left(x_{1}^{2 b_{2} b_{3} \ldots b_{n}}+x_{2}^{2 b_{1} b_{3} \ldots b_{n}}+\cdots+x_{n}^{2 b_{1} b_{2} \ldots b_{n-1}}\right)^{1 /\left(2 b_{1} b_{2} \ldots b_{n}\right)} .
$$

The function $\tau$ is a non-isotropic distance function on $\mathbb{R}^{n}$ in the sense that $\tau$ is homogeneous with respect to the non-isotropic dilations

$$
d_{t}(x)=\left(t^{b_{1}} x_{1}, t^{b_{2}} x_{2}, \ldots, t^{b_{n}} x_{n}\right)
$$

and the function $\tau(x-y)$ is a quasi-distance on $\mathbb{R}^{n}$ (as defined in [10, pp. 10-11]). In particular, $\mathbb{R}^{n}$ equipped with the quasi-distance $\tau(x-y)$ and the dilation group $\left\{d_{t}\right\}_{t>0}$ turns into a quasi-metric space of homogeneous dimension

$$
b=b_{1}+\cdots+b_{n} .
$$

The purpose of this paper is to study the singular integral operator $T$ defined initially for $f \in C_{0}^{\infty}\left(\mathbb{R}^{n}\right)$ by

$$
T f(x)=\text { p.v. } \int_{|y| \leq 1} \frac{e^{i /|y|^{\beta}}}{\tau(y)^{\alpha}} f(x-y) d y,
$$

where $\alpha$ and $\beta$ are positive parameters. If $\alpha<b$, then the kernel of $T$ is an $L^{1}$ function $\left(^{1}\right.$ ), so we assume $\alpha \geq b$ and ask how large $\beta$ should be so that, for a given $p \in(1, \infty), T$ extends to a bounded operator on $L^{p}\left(\mathbb{R}^{n}\right)$. Informally, for a given $p \in(1, \infty)$, how much oscillations do we need to

2000 Mathematics Subject Classification: 42B20, 42B10.

Key words and phrases: singular integrals, oscillatory integrals, non-isotropic singularity.

$\left({ }^{1}\right)$ This can be easily seen from Lemma 2(i) in Section 2. 
overcome a non-isotropic singularity of order $\alpha$ at the origin and obtain $L^{p}$ boundedness?

In the isotropic case, i.e. when $1=b_{1}=\cdots=b_{n}$, the operator $T$ has a long history. It was first studied in [12], where it was shown that $T$ is bounded on $L^{p}\left(\mathbb{R}^{n}\right)$ whenever

$$
\left|\frac{1}{p}-\frac{1}{2}\right|<\frac{(n / 2) \beta-\alpha+n}{n \beta},
$$

and that $T$ fails to be bounded on $L^{p}\left(\mathbb{R}^{n}\right)$ if

$$
\left|\frac{1}{p}-\frac{1}{2}\right|>\frac{(n / 2) \beta-\alpha+n}{n \beta} .
$$

The question of whether or not $T$ remains bounded on the critical spaces $L^{p}\left(\mathbb{R}^{n}\right),|1 / p-1 / 2|=((n / 2) \beta-\alpha+n) /(n \beta)$, was first considered in [2], where it was shown that $T$ is of weak type $(1,1)$ if $\alpha=n$. It was then shown in [3] that $T$ is bounded from $H^{1}\left(\mathbb{R}^{n}\right)$ to $L^{1}\left(\mathbb{R}^{n}\right)$ if $\alpha=n$. Using complex interpolation on Hardy spaces, it was also shown in [3] that if $\alpha>n$, then $T$ is bounded on the critical $L^{p}$ spaces.

The results of this paper generalize those of [12]. We show that $T$ is bounded on $L^{p}\left(\mathbb{R}^{n}\right)$ whenever

$$
\beta>4\left(b_{n}-1\right) \text { and } \quad\left|\frac{1}{p}-\frac{1}{2}\right|<\frac{(n / 2) \beta-\alpha+n}{n \beta} .
$$

The negative result was addressed in [1], where it was shown that $T$ fails to be bounded on $L^{p}\left(\mathbb{R}^{n}\right)$ whenever

$$
\beta \geq b_{n}-1 \text { and } \quad\left|\frac{1}{p}-\frac{1}{2}\right|>\frac{n \beta-2 \alpha+n+b}{2 n(1+\beta)-2 b} .
$$

Notice that when $1=b_{1}=\cdots=b_{n},(3)$ coincides with (1) and (4) coincides with (2). In the general case, however, a gap remains between the positive result in (3) and the negative result in (4). Before we carefully state our results, we would like to generalize the situation a little further.

$\mathrm{A}\left\{d_{t}\right\}$-homogeneous distance function is a continuous function $\varrho: \mathbb{R}^{n} \rightarrow$ $[0, \infty)$ satisfying $\varrho\left(d_{t} x\right)=t \varrho(x)$ for every $t>0$, and $\varrho(x) \neq 0$ for $x \neq 0$. Any $\left\{d_{t}\right\}$-homogeneous distance function $\varrho$ is comparable to $\tau$. To prove this, let $\Sigma=\left\{x \in \mathbb{R}^{n}: \tau(x)=1\right\}$ and notice that, since

$$
0<c_{1}=\inf _{\Sigma} \varrho \leq \sup _{\Sigma} \varrho=c_{2},
$$

we have

$$
c_{1} \leq \varrho(x) / \tau(x)=\varrho\left(d_{1 / \tau(x)} x\right) \leq c_{2}
$$

for $x \neq 0$. Hence

$$
c_{1} \tau(x) \leq \varrho(x) \leq c_{2} \tau(x)
$$


for all $x \in \mathbb{R}^{n}$. For later reference let us note that (5) implies

$$
c_{3}|x| \leq \varrho(x) \leq c_{4}|x|^{1 / b_{n}}
$$

for $|x| \leq 1$, and

$$
c_{3}|x|^{1 / b_{n}} \leq \varrho(x) \leq c_{4}|x|
$$

for $|x| \geq 1$, where $c_{3}=c_{1} \inf _{\mathbb{S}^{n-1}} \tau$ and $c_{4}=c_{2} \sup _{\mathbb{S}^{n-1}} \tau$. For further properties of these distance functions we refer to [11].

The results previously known about $T$ are stated in the following theorem, which was proved in [5] in dimension $n=2$ and in [1] in higher dimensions.

Theorem A. Suppose $\beta>\alpha-b \geq 0, \varrho$ is a $\left\{\delta_{t}\right\}$-homogeneous distance function which is $C^{1}$ in $\mathbb{R}^{n}-\{0\}$, and for $f \in C_{0}^{\infty}\left(\mathbb{R}^{n}\right)$ define

$$
T f(x)=\text { p.v. } \int_{|y| \leq 1} \frac{e^{i /|y|^{\beta}}}{\varrho(y)^{\alpha}} f(x-y) d y .
$$

Let

$$
Q(\alpha, \beta)=\frac{2 b+\beta-2 \alpha}{2 \beta}
$$

and

$$
Q_{j}=Q\left(\alpha, j(1+\beta)-b_{1}-\cdots-b_{j}\right), \quad j=1, \ldots, n
$$

Then:

(i) The principal value integral in (8) exists for every $x \in \mathbb{R}^{n}$.

(ii) $T$ extends to a bounded linear operator on $L^{p}\left(\mathbb{R}^{n}\right)$ whenever

$$
\left|\frac{1}{p}-\frac{1}{2}\right|<Q(\alpha, \beta)
$$

(iii) If

$$
\left|\frac{1}{p}-\frac{1}{2}\right|>\max \left[Q_{1}, \ldots, Q_{n}\right],
$$

then $T$ is not bounded on $L^{p}\left(\mathbb{R}^{n}\right)$.

Notice that, putting $b_{n+1}=\infty$, we have

$$
b_{j} \leq 1+\beta \leq b_{j+1} \Rightarrow \max \left[Q_{1}, \ldots, Q_{n}\right]=Q_{j} .
$$

In particular, if $1+\beta \leq b_{2}$, then $\max \left[Q_{1}, \ldots, Q_{n}\right]=Q_{1}=Q(\alpha, \beta)$. So we only have a sharp result (up to the end-points) in the case $1+\beta \leq b_{2}$. The main result of this paper is stated in the following theorem. 
TheOREM 1. Suppose $\beta>\alpha-b \geq 0$, $\varrho$ is a $\left\{\delta_{t}\right\}$-homogeneous distance function which is $C^{\infty}$ in $\mathbb{R}^{n}-\{0\}$, and for $f \in C_{0}^{\infty}\left(\mathbb{R}^{n}\right)$ define

$$
T f(x)=\text { p.v. } \int_{|y| \leq 1} \frac{e^{i /|y|^{\beta}}}{\varrho(y)^{\alpha}} f(x-y) d y .
$$

Then $T$ extends to a bounded linear operator on $L^{p}\left(\mathbb{R}^{n}\right)$ whenever

$$
\beta>4\left(b_{n}-1\right) \text { and }\left|\frac{1}{p}-\frac{1}{2}\right|<\frac{(n / 2) \beta-\alpha+n}{n \beta} .
$$

For further results on oscillatory strongly singular integral operators, we refer the reader to [4] and [6]-[9].

The notation $A \lesssim B$ means $A \leq C B$ for an appropriate constant $C$, and $A \approx B$ means $A \lesssim B$ and $B \lesssim A$.

2. Fourier transform estimates. Let $\varphi: \mathbb{R}^{n-1} \rightarrow \mathbb{R}$ be a $C^{\infty}$ function, and let $\Phi \in C_{0}^{\infty}\left(\mathbb{R}^{n-1}\right)$ be such that $\varphi$ has a unique critical point $x_{0} \in$ $\operatorname{supp} \Phi$. Suppose, in addition, that this critical point is non-degenerate. Then the method of stationary phase, in its most standard form (see e.g. [10]), tells us that

$$
\int e^{i \lambda \varphi(x)} \Phi(x) d x=\lambda^{-(n-1) / 2} \sum_{j=0}^{k-1} c_{j} \lambda^{-j}+O\left(\lambda^{-(n-1) / 2-k}\right)
$$

as $\lambda \rightarrow \infty$, where $c_{0}, \ldots, c_{k-1}$, as well as the constants occurring in the error term, are bounded by the $L^{\infty}$-norms of finitely many derivatives of $\varphi$ and $\Phi$. But if the $L^{2}$-norm of each derivative of $\Phi$ is much smaller than its $L^{\infty}$-norm (as is the case with the function $h_{\delta}$ defined in Lemma 2 below), then one has to adjust the standard arguments in order to obtain new satisfactory bounds. This is precisely what we plan to do in this section.

We shall use the principle of stationary phase in the following form.

Lemma 1. Let $\Phi \in C_{0}^{\infty}\left(\mathbb{R}^{n-1}\right)$ be such that

$$
\left\|D^{m} \Phi\right\|_{L^{2}\left(\mathbb{R}^{n-1}\right)} \leq C_{m} \frac{A}{\delta^{|m|}}
$$

for every multi-index $m$ in $\mathbb{R}^{n-1}$, and let $k$ be a positive integer. Then

$$
\begin{aligned}
\int e^{\pi i \lambda|x|^{2}} \Phi(x) d x= & e^{\pi i(n-1) / 4} \lambda^{-(n-1) / 2} \Phi(0)+\lambda^{-(n-1) / 2} \sum_{j=1}^{k-1} A_{j} \lambda^{-j} \\
& +A \delta^{-(n-1) / 2-2 k} O\left(\lambda^{-(n-1) / 2-k}\right)
\end{aligned}
$$

with

$$
\left|A_{j}\right| \leq \sum_{|m|=2 j}\left|D^{m} \Phi(0)\right| \quad(j=1, \ldots, k-1) .
$$


In addition to being independent of $\delta$, the constants in the error term are independent of $\Phi$ and $A$ (but, of course, depend on the $C_{m}$ 's).

Proof. Since the distributional Fourier transform of $e^{\pi i \lambda|x|^{2}}$ is

$$
e^{\pi i(n-1) / 4} \lambda^{-(n-1) / 2} e^{-\pi i \lambda^{-1}|y|^{2}},
$$

it follows that

$$
\int e^{\pi i \lambda|x|^{2}} \Phi(x) d x=e^{\pi i(n-1) / 4} \lambda^{-(n-1) / 2} \int e^{-\pi i \lambda^{-1}|y|^{2}} \widehat{\Phi}(y) d y .
$$

Also

$$
\begin{aligned}
e^{-\pi i \lambda^{-1}|y|^{2}} & =1+\sum_{j=1}^{k-1}\left(\frac{i}{4 \pi}\right)^{j} \frac{\lambda^{-j}}{j !}\left((2 \pi i)^{2}|y|^{2}\right)^{j}+O\left(\lambda^{-k}|y|^{2 k}\right) \\
& =1+\sum_{j=1}^{k-1}\left(\frac{i}{4 \pi}\right)^{j} \lambda^{-j}\left(\sum_{|m|=j} \frac{(2 \pi i y)^{2 m}}{m !}\right)+O\left(\lambda^{-k}|y|^{2 k}\right)
\end{aligned}
$$

( $m$ always denotes a multi-index in $\mathbb{R}^{n-1}$ ), so that

$$
\int e^{\pi i \lambda|x|^{2}} \Phi(x) d x=e^{\pi i(n-1) / 4} \lambda^{-(n-1) / 2} \Phi(0)+\lambda^{-(n-1) / 2} \sum_{j=1}^{k-1} A_{j} \lambda^{-j}+E(\lambda),
$$

where

$$
\begin{aligned}
A_{j} & =\left(\frac{i}{4 \pi}\right)^{j} e^{\pi i(n-1) / 4} \sum_{|m|=j} \frac{1}{m !} \int(2 \pi i y)^{2 m} \widehat{\Phi}(y) d y \\
& =\left(\frac{i}{4 \pi}\right)^{j} e^{\pi i(n-1) / 4} \sum_{|m|=j} \frac{1}{m !} \int \widehat{D^{2 m} \Phi}(y) d y \\
& =\left(\frac{i}{4 \pi}\right)^{j} e^{\pi i(n-1) / 4} \sum_{|m|=j} \frac{1}{m !} D^{2 m} \Phi(0)
\end{aligned}
$$

and

$$
|E(\lambda)| \lesssim \lambda^{-(n-1) / 2-k} \int|y|^{2 k}|\widehat{\Phi}(y)| d y .
$$

To estimate this last integral we set $\Phi_{\delta}(x)=\Phi(\delta x)$, let $N>n / 2$ be an integer, and notice that

$$
\begin{aligned}
& \int|y|^{2 k}|\widehat{\Phi}(y)| d y=\int|y|^{2 k}\left|\widehat{\Phi}_{\delta}(\delta y)\right| \delta^{n-1} d y=\delta^{-2 k} \int|v|^{2 k}\left|\widehat{\Phi}_{\delta}(v)\right| d v \\
& \leq \delta^{-2 k} \int \frac{(1+|v|)^{N+2 k}}{(1+|v|)^{N}}\left|\widehat{\Phi}_{\delta}(v)\right| d v \lesssim \delta^{-2 k} \sum_{|m| \leq N+2 k} \int \frac{1}{(1+|v|)^{N}}\left|v^{m} \widehat{\Phi}_{\delta}(v)\right| d v \\
& \approx \delta^{-2 k} \sum_{|m| \leq N+2 k} \int \frac{1}{(1+|v|)^{N}}\left|\widehat{D^{m} \Phi_{\delta}}(v)\right| d v \lesssim \delta^{-2 k} \sum_{|m| \leq N+2 k}\left\|\widehat{D^{m} \Phi_{\delta}}\right\|_{L^{2}\left(\mathbb{R}^{n-1}\right)}
\end{aligned}
$$




$$
\begin{aligned}
& =\delta^{-2 k} \sum_{|m| \leq N+2 k}\left\|D^{m} \Phi_{\delta}\right\|_{L^{2}\left(\mathbb{R}^{n-1}\right)}=\delta^{-2 k} \sum_{|m| \leq N+2 k} \delta^{|m|}\left(\int\left|D^{m} \Phi(\delta x)\right|^{2} d x\right)^{1 / 2} \\
& =\delta^{-2 k} \sum_{|m| \leq N+2 k} \delta^{|m|} \delta^{-(n-1) / 2}\left\|D^{m} \Phi\right\|_{L^{2}\left(\mathbb{R}^{n-1}\right)} \\
& \lesssim \delta^{-2 k} \sum_{|m| \leq N+2 k} A \delta^{-(n-1) / 2} \lesssim A \delta^{-(n-1) / 2-2 k} .
\end{aligned}
$$

The following is the main result in this section.

Proposition 1. Let $\psi \in C^{\infty}\left(\mathbb{R}^{n}-\{0\}\right)$ be such that

$$
\left\|D^{\nu} \psi\right\|_{L^{2}\left(\mathbb{S}^{n-1}\right)} \leq C_{\nu} \frac{A}{\delta^{|\nu|}}
$$

for every multi-index $\nu$ in $\mathbb{R}^{n}$, and let $k$ be a positive integer. Then, for $\xi=|\xi| \xi^{\prime}$ with $|\xi| \geq 1$, we have

$$
\begin{aligned}
\widehat{\psi d \sigma}(\xi)= & |\xi|^{-(n-1) / 2}\left(e^{-2 \pi i(|\xi|-(n-1) / 8)} \psi\left(\xi^{\prime}\right)+e^{2 \pi i(|\xi|-(n-1) / 8)} \psi\left(-\xi^{\prime}\right)\right) \\
& +|\xi|^{-(n-1) / 2} \sum_{j=1}^{k-1} B_{j}|\xi|^{-j} \\
& +\left(A \delta^{-(n-1) / 2-2 k}+\sum_{|\nu| \leq(n+2 k) / 2}\left\|D^{\nu} \psi\right\|_{L^{1}\left(\mathbb{S}^{n-1}\right)}\right) O\left(|\xi|^{-(n-1) / 2-k}\right),
\end{aligned}
$$

with

$$
\left|B_{j}\right| \lesssim \sum_{|\nu| \leq 2 j}\left(\left|D^{\nu} \psi\left(\xi^{\prime}\right)\right|+\left|D^{\nu} \psi\left(-\xi^{\prime}\right)\right|\right) \quad(j=1, \ldots, k-1) .
$$

Proof. We shall denote multi-indices in $\mathbb{R}^{n}$ by $\nu$ or $\nu^{\prime}$, and multi-indices in $\mathbb{R}^{n-1}$ by $m$ or $m^{\prime}$.

Notice that if $R: \mathbb{R}^{n} \rightarrow \mathbb{R}^{n}$ is a rotation with $R\left(\xi^{\prime}\right)=e_{n}=(0,0, \ldots, 1)$, then $\widehat{\psi d \sigma}(\xi)=\left(\psi \widehat{\circ R^{-1}} d \sigma\right)\left(|\xi| e_{n}\right)$ and

$$
\left|D^{\nu}\left(\psi \circ R^{-1}\right)(z)\right| \lesssim \sum_{\left|\nu^{\prime}\right|=|\nu|}\left|D^{\nu^{\prime}} \psi\left(R^{-1} z\right)\right|
$$

for all $z \in \mathbb{R}^{n}-\{0\}$. It is therefore enough to prove the theorem in the case $\xi^{\prime}=e_{n}$.

Morse's lemma tells us that there are neighborhoods $V$ and $V^{\prime}$ of 0 in $\mathbb{R}^{n-1}$ and a $C^{\infty}$ diffeomorphism $G: V \rightarrow V^{\prime}$ such that

$$
\sqrt{1-|G x|^{2}}=1-|x|^{2} / 2
$$

for all $x \in V$. Choose $0<s<1$ such that $B=B(0, s)=\left\{x \in \mathbb{R}^{n-1}:|x|<s\right\}$ $\subset V^{\prime}$ and put $U=G^{-1}(B)$. Then let $\left\{F_{1}, \ldots, F_{r}\right\}$ be a system of local 
coordinates on $\mathbb{S}^{n-1}$ where $F_{1}, F_{2}: B \rightarrow \mathbb{S}^{n-1}$ are given by

$$
F_{1}(x)=\left(x, \sqrt{1-|x|^{2}}\right) \quad \text { and } \quad F_{2}(x)=\left(x,-\sqrt{1-|x|^{2}}\right),
$$

and $F_{3}, \ldots, F_{r}$ map onto sets whose closures do not contain $\mp e_{n}$. Then

$$
\begin{aligned}
\widehat{\psi d \sigma}(\xi)= & \int_{B} e^{-2 \pi i \lambda \sqrt{1-|x|^{2}}} \psi\left(F_{1}(x)\right) q_{1}(x) d x \\
& +\int_{B} e^{2 \pi i \lambda \sqrt{1-|x|^{2}}} \psi\left(F_{2}(x)\right) q_{2}(x) d x \\
& +\sum_{l=3}^{r} \int_{\mathbb{R}^{n-1}} e^{-2 \pi i \lambda e_{n} \cdot F_{l}(x)} \psi\left(F_{l}(x)\right) q_{l}(x) d x \\
= & I_{1}(\lambda)+I_{2}(\lambda)+\sum_{l=3}^{r} I_{l}(\lambda),
\end{aligned}
$$

where $\lambda=|\xi|, q_{1}, \ldots, q_{r}$ are real-valued $C^{\infty}$ functions, $q_{1}$ is supported in $B$ and $q_{1}(0)=1, q_{2}=q_{1}$, and $\operatorname{supp} q_{l} \subset$ Domain $F_{l}$.

The phase function of each of the integrals $I_{l}(3 \leq l \leq r)$ has no critical points in $\operatorname{supp} q_{l}$. Thus standard non-stationary phase estimates tell us that

$$
\left|I_{l}\right| \lesssim \lambda^{-N} \sum_{|m| \leq N}\left\|D^{m} \eta_{l}\right\|_{L^{1}\left(\mathbb{R}^{n-1}\right)}
$$

for every positive integer $N$, where $\eta_{l}=\left(\psi \circ F_{l}\right) q_{l}$ (this is essentially the inequality $|\widehat{f}(y)| \lesssim(1+|y|)^{-N} \sum_{|m| \leq N}\left\|D^{m} f\right\|_{L^{1}\left(\mathbb{R}^{n-1}\right)}$ applied after an appropriate partition of unity on $\left.\operatorname{supp} q_{l}\right)$. Taking $N=\lfloor(n+2 k) / 2\rfloor$, we get

$$
\left|I_{l}\right| \lesssim \lambda^{-(n-1) / 2-k} \sum_{|m| \leq(n+2 k) / 2}\left\|D^{m} \eta_{l}\right\|_{L^{1}\left(\mathbb{R}^{n-1}\right)}
$$

Now

$$
\left|D^{m} \eta_{l}(x)\right| \lesssim \sum_{\left|m^{\prime}\right| \leq|m|}\left|D^{m^{\prime}}\left(\psi \circ F_{l}\right)(x)\right| \lesssim \sum_{|\nu| \leq|m|}\left|D^{\nu} \psi\left(F_{l}(x)\right)\right|
$$

for all $x \in \mathbb{R}^{n-1}$, and it follows that

$$
\left\|D^{m} \eta_{l}\right\|_{L^{1}\left(\mathbb{R}^{n-1}\right)} \lesssim \sum_{|\nu| \leq|m|}\left\|D^{\nu} \psi\right\|_{L^{1}\left(\mathbb{S}^{n-1}\right)}
$$

Therefore

$$
\left|I_{l}\right| \lesssim \lambda^{-(n-1) / 2-k} \sum_{|\nu| \leq(n+2 k) / 2}\left\|D^{\nu} \psi\right\|_{L^{1}\left(\mathbb{S}^{n-1}\right)}
$$

for $l=3, \ldots, r$. It remains to estimate $I_{1}$ and $I_{2}$. 
We start by looking at $I_{1}$. Making the change of variables $u=G^{-1} x$ and using (9), we get

$$
I_{1}=e^{-2 \pi i \lambda} \int_{U} e^{\pi i \lambda|u|^{2}} \Psi(u) q(u) d u
$$

where $\Psi=\psi \circ F_{1} \circ G$ and $q(u)=q_{1}(G u)\left|J_{G}(u)\right|$. Observe that

$$
\left|D^{m} \Psi(u)\right| \lesssim \sum_{|\nu| \leq|m|}\left|\left(D^{\nu} \psi\right) \circ F_{l} \circ G(u)\right|
$$

for all $u \in U$. Set $\Phi=q \Psi$. Then $\Phi \in C_{0}^{\infty}(U), \Phi(0)=\psi\left(e_{n}\right)\left(\left|J_{G}(0)\right|=1\right.$ by (9)), and

$$
\left|D^{m} \Phi(u)\right| \lesssim \sum_{|\nu| \leq|m|}\left|\left(D^{\nu} \psi\right) \circ F_{l} \circ G(u)\right|
$$

for all $u \in U$. This last inequality implies that

$$
\left\|D^{m} \Phi\right\|_{L^{p}\left(\mathbb{R}^{n-1}\right)} \lesssim \sum_{|\nu| \leq|m|}\left\|D^{\nu} \psi\right\|_{L^{p}\left(\mathbb{S}^{n-1}\right)}
$$

for $1 \leq p \leq \infty$. In particular,

$$
\left\|D^{m} \Phi\right\|_{L^{2}\left(\mathbb{R}^{n-1}\right)} \lesssim \sum_{|\nu| \leq|m|} C_{\nu} \frac{A}{\delta^{|\nu|}} \leq C_{m} \frac{A}{\delta^{|m|}}
$$

Applying Lemma 1, we get

$$
\begin{aligned}
I_{1}= & e^{-2 \pi i \lambda} \int e^{\pi i \lambda|u|^{2}} \Phi(u) d u \\
= & \lambda^{-(n-1) / 2} e^{-2 \pi i(\lambda-(n-1) / 8)} \psi\left(e_{n}\right)+\lambda^{-(n-1) / 2} \sum_{j=1}^{k-1} A_{j} \lambda^{-j} \\
& +A \delta^{-(n-1) / 2-2 k} O\left(\lambda^{-(n-1) / 2-k}\right)
\end{aligned}
$$

with

$$
\left|A_{j}\right| \leq \sum_{|m|=2 j}\left|D^{m} \Phi(0)\right| \lesssim \sum_{|\nu| \leq 2 j}\left|\left(D^{\nu} \psi\right) \circ F_{l} \circ G(0)\right|=\sum_{|\nu| \leq 2 j}\left|D^{\nu} \psi\left(e_{n}\right)\right| .
$$

Since $\bar{I}_{2}$ is the same as $I_{1}$ with $\psi \circ F_{1}$ replaced by $\bar{\psi} \circ F_{2}$, it follows that

$$
\begin{aligned}
I_{1}+I_{2}= & \lambda^{-(n-1) / 2}\left(e^{-2 \pi i(\lambda-(n-1) / 8)} \psi\left(e_{n}\right)+e^{2 \pi i(\lambda-(n-1) / 8)} \psi\left(-e_{n}\right)\right) \\
& +\lambda^{-(n-1) / 2} \sum_{j=1}^{k-1} B_{j} \lambda^{-j}+A \delta^{-(n-1) / 2-2 k} O\left(\lambda^{-(n-1) / 2-k}\right)
\end{aligned}
$$

with

$$
\left|B_{j}\right| \lesssim \sum_{|\nu| \leq 2 j}\left(\left|D^{\nu} \psi\left(e_{n}\right)\right|+\left|D^{\nu} \psi\left(-e_{n}\right)\right|\right) .
$$

This combined with (10) gives the desired result. 
We are now in a position to describe the asymptotic behavior at infinity of the Fourier transforms of the measures $\varrho(r \theta)^{-\alpha} d \sigma(\theta)$, but we first need the following lemma.

Lemma 2. Let $\varrho$ be a $\left\{d_{t}\right\}$-homogeneous distance function which is $C^{\infty}$ in $\mathbb{R}^{n}-\{0\}$, and let $\alpha>b-1=b_{2}+\cdots+b_{n}$. For $0<\delta \leq 1$ and $x \in \mathbb{R}^{n}-\{0\}$, set

$$
h(x)=\varrho(x)^{-\alpha} \quad \text { and } \quad h_{\delta}(x)=h(\delta x) .
$$

Then:

(i) $\left\|h_{\delta}\right\|_{L^{p}\left(\mathbb{S}^{n-1}\right)} \approx \delta^{(b-n) / p-\alpha}$ for $1 \leq p \leq \infty$.

(ii) $\left|d^{j}\left(h_{\delta}(\theta)\right) / d \delta^{j}\right| \lesssim h_{\delta}(\theta) / \delta^{j}$ for all $\theta \in \mathbb{S}^{n-1}, j=1,2, \ldots$

(iii) $\int_{\mathbb{S}^{n-1}}\left|d^{j}\left(h_{\delta}(\theta)\right) / d \delta^{j}\right| d \sigma(\theta) \lesssim \delta^{b-n-\alpha-j}, j=1,2, \ldots$.

(iv) If $\nu$ is a multi-index in $\mathbb{R}^{n}$, then

$$
\left|D^{\nu} h_{\delta}(\omega)\right| \lesssim \delta^{-|\nu|\left(b_{n}-1\right)} h_{\delta}(\omega) \quad \text { for all } \omega \in \mathbb{S}^{n-1} .
$$

(v) If $\nu$ is a multi-index in $\mathbb{R}^{n}$, then

$$
\left\|D^{\nu} h_{\delta}\right\|_{L^{p}\left(\mathbb{S}^{n-1}\right)} \lesssim \delta^{-|\nu|\left(b_{n}-1\right)} \delta^{(b-n) / p-\alpha} \quad \text { for } 1 \leq p \leq \infty .
$$

Proof. For the proof of (i)-(iii) we refer the reader to [1].

(iv) Since $h(x)=t^{\alpha} h\left(d_{t} x\right)$, it follows that

$$
D^{\nu} h(x)=t^{\alpha+\nu \cdot\left(b_{1}, \ldots, b_{n}\right)} D^{\nu} h\left(d_{t} x\right) .
$$

Putting $t=1 / \varrho(x)$, we get $\left|D^{\nu} h(x)\right| \lesssim \varrho(x)^{-\alpha-\nu \cdot\left(b_{1}, \ldots, b_{n}\right)}$, and hence

$$
\left|D^{\nu} h_{\delta}(x)\right| \lesssim \delta^{|\nu|} \varrho(\delta x)^{-\alpha-\nu \cdot\left(b_{1}, \ldots, b_{n}\right)}=\delta^{|\nu|} \varrho(\delta x)^{-\nu \cdot\left(b_{1}, \ldots, b_{n}\right)} h_{\delta}(x)
$$

for $x \neq 0$. So for $\omega \in \mathbb{S}^{n-1}$, (6) gives

$$
\left|D^{\nu} h_{\delta}(\omega)\right| \lesssim \frac{\delta^{|\nu|}}{\delta^{\nu \cdot\left(b_{1}, \ldots, b_{n}\right)}} h_{\delta}(\omega) \lesssim \frac{\delta^{|\nu|}}{\delta^{|\nu| b_{n}}} h_{\delta}(\omega),
$$

where we have used the standing assumption $1=b_{1} \leq \cdots \leq b_{n}$.

(v) follows from (i) and (iii).

Proposition 2. Let $\varrho, \alpha, h$, and $h_{\delta}$ be as in Lemma 2. If $k$ is a positive integer, then, for $\xi=|\xi| \xi^{\prime},|\xi| \geq 1$,

$$
\begin{aligned}
\widehat{h_{\delta} d \sigma}(\xi)= & \frac{h_{\delta}\left(\xi^{\prime}\right)}{|\xi|^{(n-1) / 2}} e^{-2 \pi i(|\xi|-(n-1) / 8)}+\frac{h_{\delta}\left(-\xi^{\prime}\right)}{|\xi|^{(n-1) / 2}} e^{2 \pi i(|\xi|-(n-1) / 8)} \\
& +\frac{h_{\delta}\left(\xi^{\prime}\right)+h_{\delta}\left(-\xi^{\prime}\right)}{2|\xi|^{(n-1) / 2}} \sum_{j=1}^{k-1} \frac{C_{j}}{\delta^{2 j\left(b_{n}-1\right)}|\xi|^{j}} \\
& +\frac{\delta^{(b+4 k-1) / 2}}{\delta^{\alpha+(n+4 k-1) b_{n} / 2}} O\left(\frac{1}{|\xi|^{(n-1) / 2+k}}\right)
\end{aligned}
$$

with $\left|C_{j}\right| \lesssim 1(j=1, \ldots, k-1)$. 
Proof. By Lemma 2(v),

$$
\left\|D^{\nu} h_{\delta}\right\|_{L^{2}\left(\mathbb{S}^{n-1}\right)} \leq C_{\nu} \frac{\delta^{(b-n) / 2-\alpha}}{\delta^{|\nu|\left(b_{n}-1\right)}}
$$

for every multi-index $\nu$, and

$$
\sum_{|\nu| \leq(n+2 k) / 2}\left\|D^{\nu} h_{\delta}\right\|_{L^{1}\left(\mathbb{S}^{n-1}\right)} \lesssim \sum_{|\nu| \leq(n+2 k) / 2} \frac{\delta^{b-n-\alpha}}{\delta^{|\nu|\left(b_{n}-1\right)}} \lesssim \frac{\delta^{b-n / 2+k}}{\delta^{\alpha+(n+2 k) b_{n} / 2}} .
$$

Applying Proposition 1 with $\psi=h_{\delta}, A=\delta^{(b-n) / 2-\alpha}$, and $\delta$ replaced by $\delta^{b_{n}-1}$, we get

$$
\begin{aligned}
\widehat{h_{\delta} d \sigma}(\xi)= & \frac{h_{\delta}\left(\xi^{\prime}\right)}{|\xi|^{(n-1) / 2}} e^{-2 \pi i(|\xi|-(n-1) / 8)}+\frac{h_{\delta}\left(-\xi^{\prime}\right)}{|\xi|^{(n-1) / 2}} e^{2 \pi i(|\xi|-(n-1) / 8)} \\
& +\frac{1}{|\xi|^{(n-1) / 2}} \sum_{j=1}^{k-1} \frac{B_{j}}{|\xi|^{j}} \\
& +\left(\frac{\delta^{(b-n) / 2-\alpha}}{\delta^{\left(b_{n}-1\right)((n-1) / 2+2 k)}}+\frac{\delta^{b-n / 2+k}}{\delta^{\alpha+(n+2 k) b_{n} / 2}}\right) O\left(\frac{1}{|\xi|^{(n-1) / 2+k}}\right),
\end{aligned}
$$

with

$$
\begin{aligned}
\left|B_{j}\right| & \lesssim \sum_{|\nu| \leq 2 j}\left(\left|D^{\nu} h_{\delta}\left(\xi^{\prime}\right)\right|+\left|D^{\nu} h_{\delta}\left(-\xi^{\prime}\right)\right|\right) \\
& \lesssim \sum_{|\nu| \leq 2 j} \frac{h_{\delta}\left(\xi^{\prime}\right)+h_{\delta}\left(-\xi^{\prime}\right)}{\delta^{|\nu|\left(b_{n}-1\right)}} \lesssim \frac{h_{\delta}\left(\xi^{\prime}\right)+h_{\delta}\left(-\xi^{\prime}\right)}{\delta^{2 j\left(b_{n}-1\right)}},
\end{aligned}
$$

where we used Lemma 2(iv). To complete the proof, notice that

$$
\frac{\delta^{b-n / 2+k}}{\delta^{\alpha+(n+2 k) b_{n} / 2}} \leq \frac{\delta^{(b-n) / 2-\alpha}}{\delta^{\left(b_{n}-1\right)((n-1) / 2+2 k)}}=\frac{\delta^{(b+4 k-1) / 2}}{\delta^{\alpha+(n+4 k-1) b_{n} / 2}}
$$

because $b-n+(2 k-1)\left(b_{n}-1\right) \geq 0$.

3. Proof of Theorem 1. Our method of proof follows the general lines of [9]. In particular, we shall use integration by parts in the following form, which is Lemma 1 in [9].

Lemma 3. Suppose $I$ is an open interval, $g \in C_{0}^{\infty}(I), F \in C^{\infty}(I), F$ is real-valued, $F^{\prime} \neq 0$ on $I$, and $k$ is a positive integer. Then

$$
\int_{I} g(r) e^{i F(r)} d r=\int_{I} G_{k}(r) e^{i F(r)} d r,
$$

where $G_{k}$ is a linear combination of functions of the form

$$
\left(g^{(s)}\right)\left(F^{\prime}\right)^{-k-\mu} \prod_{q=1}^{\mu} F^{\left(j_{q}\right)}
$$

with $0 \leq s \leq k, 0 \leq \mu \leq k$, and $2 \leq j_{q} \leq k+1$. 
For $y \in \mathbb{R}^{n}-\{0\}$, define

$$
\widetilde{K}(y)= \begin{cases}h(y) e^{i /|y|^{\beta}} & \text { if } 0<|y| \leq 1, \\ 0 & \text { if }|y|>1 .\end{cases}
$$

Here, and for the rest of this paper, $h$ and $h_{\delta}$ are the functions defined in Lemma 2. Also, let $\eta \in C_{0}^{\infty}\left(\mathbb{R}^{n}\right)$ be radial, non-negative, supported in the ring $1 / 2<|y|<1$, and satisfying

$$
\sum_{l=0}^{\infty} \eta_{l}(y)= \begin{cases}1 & \text { if } 0<|y| \leq 1 \\ 0 & \text { if }|y| \geq 2\end{cases}
$$

where $\eta_{l}(y)=\eta\left(2^{l} y\right)$. Then $\widetilde{K}=\sum_{l=0}^{\infty} K_{l}$, where $K_{l}=\eta_{l} \widetilde{K}$. Clearly, $K_{0} \in$ $L^{1}\left(\mathbb{R}^{n}\right)$. On the other hand, if $k$ and $l$ are positive integers and $\psi \in \mathcal{S}\left(\mathbb{R}^{n}\right)$, then

$$
\int K_{l}(y) \psi(y) d y=2^{-n l} \int_{\mathbb{S}^{n-1}} \int_{1 / 2}^{2} g(r) e^{i F(r)} d r d \sigma(\theta)
$$

with $F(r)=2^{l \beta} r^{-\beta}$ and $g(r)=\eta(r) h_{2^{-l} r}(\theta) \psi\left(2^{-l} r \theta\right) r^{n-1}$, and so by Lemma 3 ,

$$
\begin{aligned}
\left|\int K_{l}(y) \psi(y) d y\right| & \lesssim 2^{-n l} \int_{\mathbb{S}^{n-1}} \int_{1 / 2}^{2} \sum_{s=0}^{k}\left|g^{(s)}(r)\right| 2^{-k l \beta} d r d \sigma(\theta) \\
& \lesssim 2^{-l(k \beta+n)}\|\psi\|_{C^{k}} \int_{\mathbb{S}^{n-1}} \int_{1 / 2}^{2} \sum_{j=0}^{k}\left|\frac{d^{j}\left(h_{2^{-l} r}(\theta)\right)}{d r^{j}}\right| d r d \sigma(\theta) \\
& \lesssim 2^{-l(k \beta-\alpha+b)}\|\psi\|_{C^{k}},
\end{aligned}
$$

where in the last line we have used Lemma 2(iii). Since the implicit constants are independent of $l$, we can choose $k$ large enough for $\sum_{l=1}^{\infty} 2^{-l(k \beta-\alpha+b)} \lesssim 1$ and conclude that the mapping

$$
\psi \mapsto \sum_{l=1}^{\infty} \int K_{l}(y) \psi(y) d y
$$

defines a tempered distribution $K$ such that $K_{0}+K$ agrees with $\widetilde{K}$ away from the origin, $K_{0}+K=$ p.v. $\widetilde{K}$ when $\beta>\alpha-b$, and $\widehat{K}=\sum_{l=1}^{\infty} \widehat{K}_{l}$ in $\mathcal{S}^{\prime}\left(\mathbb{R}^{n}\right)$. It is therefore enough to prove the theorem for the operator $\widetilde{T}=K * f$. We shall start by estimating $\widehat{K}$.

Fix a positive integer $l$, a point $\xi \in \mathbb{R}^{n}$, a small constant $c$ and a large constant $C$. We are going to estimate $\widehat{K}_{l}(\xi)$ in three different ways depending on whether $2^{-l} \leq c|\xi|^{-1 /(\beta+1)}$ (this includes the possibility $\xi=0$ ), $c|\xi|^{-1 /(\beta+1)}<2^{-l}<\bar{C}|\xi|^{-1 /(\beta+1)}$, or $C|\xi|^{-1 /(\beta+1)} \leq 2^{-l}$. 
Suppose $2^{-l} \leq c|\xi|^{-1 /(\beta+1)}$. Then

$$
\widehat{K}_{l}(\xi)=\int e^{-2 \pi i \xi \cdot y} K_{l}(y) d y=2^{-n l} \int_{\mathbb{S}^{n-1}} \int_{1 / 2}^{2} g_{l}(r) e^{i F_{l}(r)} d r d \sigma(\theta)
$$

with $g_{l}(r)=\eta(r) h_{2^{-l} r}(\theta) r^{n-1}$ and $F_{l}(r)=2^{l \beta} r^{-\beta}-2 \pi 2^{-l} r(\xi \cdot \theta)$. Since

$$
\left|F_{l}^{\prime}(r)\right| \geq \beta \frac{2^{l \beta}}{r^{\beta+1}}-2 \pi 2^{-l}|\xi| \geq \beta \frac{2^{l \beta}}{2^{\beta+1}}-2 \pi c^{\beta+1} 2^{l \beta}
$$

for $1 / 2<r<2, c$ must be small enough for one to have

$$
\left|F_{l}^{\prime}(r)\right| \gtrsim 2^{l \beta} \quad \text { for } 1 / 2<r<2 .
$$

By Lemma 3, we then have

$$
\begin{aligned}
\left|\int_{1 / 2}^{2} g_{l}(r) e^{i F_{l}(r)} d r\right| & \lesssim \sum_{s=0}^{k} \sum_{\mu=0}^{k} \int_{1 / 2}^{2}\left|g_{l}^{(s)}(r)\right| 2^{l \beta(-k-\mu)} \prod_{q=1}^{\mu} 2^{l \beta} d r \\
& \lesssim 2^{-l \beta k} \sum_{s=0}^{k} \int_{1 / 2}^{2}\left|g_{l}^{(s)}(r)\right| d r
\end{aligned}
$$

and it follows from Lemma 2(iii) that

$$
\left|\widehat{K}_{l}(\xi)\right| \lesssim 2^{-l(k \beta-\alpha+b)} .
$$

Before we consider the remaining two cases, let us write

$$
\widehat{K}_{l}(\xi)=2^{-n l} \int_{1 / 2}^{2} e^{i 2^{l \beta} / r^{\beta}} \eta(r) r^{n-1} \widehat{h_{\delta} d \sigma}(\delta \xi) d r,
$$

where $\delta=2^{-l} r$. Notice that by $(5), h_{\delta}(\theta) \approx h_{\delta}(-\theta)$ and $h_{\delta}(\theta) \approx h_{2^{-l}}(\theta)$ for all $1 / 2<r<2$ and $\theta \in \mathbb{S}^{n-1}$. By Proposition 2 , we now have

$$
\widehat{K}_{l}(\xi)=J_{1}+J_{2}+E_{1}+E_{2},
$$

where

$$
J_{1}=\frac{2^{-l(n+1) / 2}}{|\xi|^{(n-1) / 2}} \int \eta(r) r^{(n-1) / 2} h_{\delta}\left(\xi^{\prime}\right) e^{i \phi_{l}(r)} d r
$$

with

$$
\phi_{l}(r)=\frac{2^{l \beta}}{r^{\beta}}-2 \pi\left(2^{-l} r|\xi|-\frac{n-1}{8}\right),
$$

$J_{2}$ is the same as $J_{1}$ but with $\xi^{\prime}$ replaced by $-\xi^{\prime}$ and $\phi_{l}(r)$ modified by a 
sign,

$$
\begin{aligned}
\left|E_{1}\right| & \lesssim \frac{2^{-l(n+1) / 2}}{|\xi|^{(n-1) / 2}} \sum_{j=1}^{k-1} \frac{1}{|\xi|^{j}} \int \eta(r) r^{(n-1) / 2} \frac{h_{\delta}\left(\xi^{\prime}\right)}{\delta^{2 j\left(b_{n}-1\right) \delta^{j}}} d r \\
& \lesssim \frac{2^{-l(n+1) / 2}}{|\xi|^{(n-1) / 2}} h_{2^{-l}}\left(\xi^{\prime}\right) \sum_{j=1}^{k-1} \frac{2^{l\left(2 b_{n}-1\right) j}}{\mid \xi^{j}},
\end{aligned}
$$

and

$$
\left|E_{2}\right| \lesssim \frac{2^{l q_{k}}}{|\xi|^{k+(n-1) / 2}}
$$

with

$$
q_{k}=\alpha+\frac{n+4 k-1}{2} b_{n}-\frac{b+4 k-1}{2}-\frac{n+1}{2}+k>0
$$

(because $\alpha \geq b \geq n$ ). Recall that by $(6), h_{2^{-l}}\left(\xi^{\prime}\right) \lesssim 2^{l \alpha}$, so

$$
\left|E_{1}\right| \lesssim \frac{2^{l(\alpha-(n+1) / 2)}}{|\xi|^{(n-1) / 2}} \sum_{j=1}^{k-1} \frac{2^{l\left(2 b_{n}-1\right) j}}{|\xi|^{j}} .
$$

Suppose $c|\xi|^{-1 /(\beta+1)}<2^{-l}<C|\xi|^{-1 /(\beta+1)}$. Using (12) and (13), we see that

$$
\left|E_{1}\right| \lesssim 2^{-l((n-1) \beta / 2-\alpha+n)} \sum_{j=1}^{k-1} 2^{-l\left(\beta-2 b_{n}+2\right) j} \lesssim 2^{-l(n \beta / 2-\alpha+n)}
$$

provided $\beta \geq 4\left(b_{n}-1\right)$, and

$$
\left|E_{2}\right| \lesssim 2^{-l(n \beta / 2-\alpha+n)}
$$

provided $k$ is large enough for

$$
(\beta+1)\left(\frac{n-1}{2}+k\right)-q_{k} \geq \frac{n}{2} \beta-\alpha+n
$$

to hold. We now consider $J_{1}$. Since $\left|\phi_{l}^{\prime \prime}(r)\right| \gtrsim 2^{l \beta}$ for $1 / 2<r<2$, van der Corput's lemma (see [10, pp. 332-334]) tells us that

$$
\begin{aligned}
\left|J_{1}\right| & \lesssim \frac{2^{-l(n+1) / 2}}{|\xi|^{(n-1) / 2}} 2^{-l \beta / 2} \int\left|\frac{d}{d r}\left(\eta(r) r^{(n-1) / 2} h_{\delta}\left(\xi^{\prime}\right)\right)\right| d r \\
& \lesssim \frac{2^{-l(n+1+\beta) / 2}}{|\xi|^{(n-1) / 2}} \int\left(h_{\delta}\left(\xi^{\prime}\right)+2^{-l} \delta^{-1} h_{\delta}\left(\xi^{\prime}\right)\right) d r
\end{aligned}
$$

where we have used Lemma 2(ii). Hence

$$
\left|J_{1}\right| \lesssim \frac{2^{-l(n+1+\beta) / 2}}{|\xi|^{(n-1) / 2}} h_{2^{-l}}\left(\xi^{\prime}\right) \lesssim 2^{-l(n \beta / 2-\alpha+n)} .
$$


Similarly,

$$
\left|J_{2}\right| \lesssim 2^{-l(n \beta / 2-\alpha+n)} .
$$

Combining (14)-(16), and (17), we get

$$
\left|\widehat{K_{l}(\xi)}\right| \lesssim 2^{-l(n \beta / 2-\alpha+n)}
$$

provided $\beta \geq 4\left(b_{n}-1\right)$.

Suppose $C|\xi|^{-1 /(\beta+1)} \leq 2^{-l}$. Then (13) implies

$$
\begin{aligned}
\left|E_{1}\right| & \lesssim \frac{2^{l(\alpha-(n+1) / 2)}}{|\xi|^{(n-1) / 2}} \sum_{j=1}^{k-1}|\xi|^{\left(\left(2 b_{n}-1\right) /(\beta+1)-1\right) j} \\
& \lesssim 2^{l(\alpha-(n+1) / 2)}|\xi|^{-\left((n+1) \beta+n-4 b_{n}+3\right) /(2(\beta+1))}
\end{aligned}
$$

provided $\beta \geq 2\left(b_{n}-1\right)$. This time we use (19) instead of (14), (12) instead of (15), and we replace (16) and (17) by the better estimates

$$
\begin{aligned}
\left|J_{1}\right|,\left|J_{2}\right| & \lesssim \frac{2^{-l(n+1) / 2}}{|\xi|^{(n-1) / 2}}|\xi|^{-\beta /(\beta+1)} h_{2^{-l}}\left(\xi^{\prime}\right) \\
& \lesssim 2^{l(\alpha-(n+1) / 2)}|\xi|^{-((n+1) \beta+n-1) /(2(\beta+1))}
\end{aligned}
$$

(which follows from van der Corput's lemma after noticing that now $\left|\phi_{l}^{\prime}(r)\right|$ $\gtrsim|\xi|^{\beta /(\beta+1)}$ for all $1 / 2<r<2$, provided $C$ is large enough) to get

$$
\begin{aligned}
\left|\widehat{K}_{l}(\xi)\right| \lesssim & 2^{l(\alpha-(n+1) / 2)}|\xi|^{-((n+1) \beta+n-1) /(2(\beta+1))} \\
& +2^{l(\alpha-(n+1) / 2)}|\xi|^{-\left((n+1) \beta+n-4 b_{n}+3\right) /(2(\beta+1))}+\frac{2^{l q_{k}}}{|\xi|^{k+(n-1) / 2}}
\end{aligned}
$$

provided $\beta \geq 2\left(b_{n}-1\right)$.

We now have all we need to estimate $\widehat{K}(\xi)$. If $|\xi| \leq c^{\beta+1}$, (11) tells us that

$$
\sum_{l=1}^{\infty}\left|\widehat{K}_{l}(\xi)\right| \lesssim 1
$$

with no conditions on $\beta$ except being positive (of course, $k$ has to be chosen large enough for $k \beta-\alpha+b>0$ ). If $|\xi|>c^{\beta+1}$, then (11), (18), and (21) tell us that

$$
\begin{aligned}
\sum_{l=1}^{\infty}\left|\widehat{K}_{l}(\xi)\right| \lesssim & |\xi|^{-(k \beta-\alpha+b) /(\beta+1)}+|\xi|^{-((n / 2) \beta-\alpha+b) /(\beta+1)} \\
& +|\xi|^{-((n+1) \beta-2 \alpha+2 n) /(2(\beta+1))} \\
& +|\xi|^{-\left((n+1) \beta-2 \alpha+2 n-4 b_{n}+4\right) /(2(\beta+1))} \\
& +|\xi|^{-\left((n-1-2 k) \beta-2 \alpha+2 n-(n+4 k-1) b_{n}-1\right) /(2(\beta+1))} \\
\lesssim & |\xi|^{-(n \beta-2 \alpha+2 n) /(2(\beta+1))}
\end{aligned}
$$


provided $\beta \geq 4\left(b_{n}-1\right)$ and $k$ is large. Thus

$$
\sum_{l=1}^{\infty}\left|\widehat{K}_{l}(\xi)\right| \lesssim(1+|\xi|)^{-(n \beta-2 \alpha+2 n) /(2(\beta+1))}
$$

for all $\xi \in \mathbb{R}^{n}$, provided $\beta \geq 4\left(b_{n}-1\right)$. Therefore, if $\beta \geq 4\left(b_{n}-1\right)$ and $(n / 2) \beta-\alpha+n \geq 0$, then $\widehat{K}$ is a function and

$$
|\widehat{K}(\xi)| \lesssim(1+|\xi|)^{-(n \beta-2 \alpha+2 n) /(2(\beta+1))}
$$

for all $\xi \in \mathbb{R}^{n}$.

To establish the $L^{p}$ inequality, we shall use complex interpolation. Choose a number $a$ such that $\alpha-(n / 2) \beta \leq a<n$, and for $z=u+i v \in \mathbb{C}$, set

$$
M_{z}(y)=\varrho(y)^{\alpha+(n / 2)(z-1) \beta-a} K(y) .
$$

We consider the analytic family $\left\{R_{z}\right\}_{0 \leq u \leq 1}$ of operators defined on the domain of Schwartz functions by

$$
R_{z} f=M_{z} * f .
$$

Clearly,

$$
R_{((n / 2) \beta+a-\alpha) /((n / 2) \beta)}=\widetilde{T} .
$$

If $u=1$, then $\operatorname{Re}(a-(n / 2)(z-1) \beta)=a<n$, so $M_{1+i v} \in L^{1}\left(\mathbb{R}^{n}\right)$ with $L^{1}$-norm independent of $v$. Thus

$$
\left\|R_{1+i v} f\right\|_{L^{1}\left(\mathbb{R}^{n}\right)} \lesssim\|f\|_{L^{1}\left(\mathbb{R}^{n}\right)} .
$$

Our next goal is to show that $R_{i v}$ satisfies an $L^{2}$ inequality with a constant having polynomial growth in $v$. Equivalently, we need to show that the Fourier transform of $M_{i v}$ is a function which is bounded by $C(1+|v|)^{N}$ for some constants $C$ and $N$. Following the same steps that led to (22), we see that

$$
\begin{aligned}
\left|\widehat{M}_{i v}(\xi)\right| & \lesssim(1+|v|)^{N}(1+|\xi|)^{-(n \beta-2(a+(n / 2) \beta)+2 n) /(2(\beta+1))} \\
& =(1+|v|)^{N}(1+|\xi|)^{(a-n) /(\beta+1)} \leq(1+|v|)^{N}
\end{aligned}
$$

for all $\xi \in \mathbb{R}^{n}$. Thus

$$
\left\|R_{i v} f\right\|_{L^{2}\left(\mathbb{R}^{n}\right)} \lesssim(1+|v|)^{N}\|f\|_{L^{2}\left(\mathbb{R}^{n}\right)} .
$$

Interpolating between the $L^{1}$ inequality (23) and the $L^{2}$ inequality (24), we conclude that

$$
\left\|R_{u} f\right\|_{L^{p}\left(\mathbb{R}^{n}\right)} \lesssim\|f\|_{L^{p}\left(\mathbb{R}^{n}\right)}
$$

whenever $0 \leq u \leq 1$ and $1 / p=(1-u) / 2+u=u / 2$. So

$$
\|\widetilde{T} f\|_{L^{p}\left(\mathbb{R}^{n}\right)} \lesssim\|f\|_{L^{p}\left(\mathbb{R}^{n}\right)}
$$

whenever

$$
\alpha-(n / 2) \beta \leq a<n \quad \text { and } \quad \frac{1}{p}-\frac{1}{2}=\frac{(n / 2) \beta+a-\alpha}{n \beta},
$$


and so (25) holds whenever

$$
0 \leq \frac{1}{p}-\frac{1}{2}<\frac{(n / 2) \beta-\alpha+n}{n \beta} .
$$

Finally, a duality argument shows that (25) holds whenever

$$
\left|\frac{1}{p}-\frac{1}{2}\right|<\frac{(n / 2) \beta-\alpha+n}{n \beta},
$$

as desired.

\section{References}

[1] F. Abi-Khuzam and B. Shayya, A class of non-homogeneous singular integrals in $\mathbb{R}^{n}$, J. Math. Anal. Appl. 50 (2001), 1015-1025.

[2] C. Fefferman, Inequalities for strongly singular convolution operators, Acta Math. 124 (1970), 9-36.

[3] C. Fefferman and E. M. Stein, $H^{p}$ spaces of several variables, ibid. 129 (1972), 137-193.

[4] N. Lyall, Strongly singular convolution operators on the Heisenberg group, Trans. Amer. Math. Soc., to appear.

[5] B. Shayya, Singular integrals with nonhomogeneous oscillatory kernels, Indiana Univ. Math. J. 50 (2001), 1015-1025.

[6] —, Nonisotropic strongly singular integral operators, Trans. Amer. Math. Soc. 354 (2002), 4893-4907.

[7] P. Sjölin, $L^{p}$ estimates for strongly singular convolution operators in $\mathbb{R}^{n}$, Ark. Mat. 14 (1976), 59-64.

[8] -, An $H^{p}$ inequality for strongly singular integrals, Math. Z. 165 (1979), 231-238.

[9] -, Convolution with oscillating kernels, Indiana Univ. Math. J. 30 (1981), 47-56.

[10] E. M. Stein, Harmonic Analysis: Real-Variable Methods, Orthogonality, and Oscillatory Integrals, Princeton Univ. Press, 1993.

[11] E. M. Stein and S. Wainger, Problems in harmonic analysis related to curvature, Bull. Amer. Math. Soc. 84 (1978), 1239-1295.

[12] S. Wainger, Special trigonometric series in $k$ dimensions, Mem. Amer. Math. Soc. 59 (1965).

Department of Mathematics

American University of Beirut

Beirut, Lebanon

E-mail: bshayya@aub.edu.lb

Received February 12, 2007

Revised version April 10, 2008 